\section{Planificación en redes de área local inalámbricas en escenarios internos: elementos, herramientas y cuestiones prácticas}

Antonio Cortés

Universidad de Panamá

antonio.cortes@up.ac.pa

DOI https://doi.org/10.33412/pri.v9.1.2062

\section{(2)(1)(2)}

Resumen: En la actualidad unas de las dificultades que presentan las redes inalámbricas de área local (WLAN) en espacios internos, por ejemplo, edificios, oficinas, distribución de cubículos entre pisos, es la adecuada cobertura a la cual tiene acceso diversos tipos de artefactos como son los celulares, laptops, computadoras, a través de la conexión inalámbrica que provee los diversos puntos de acceso (AP) distribuidos en diversos espacios por donde transitan o trabajan las personas. En este artículo presentamos un análisis de los escenarios internos, al considerar una serie de elementos básicos, características, herramientas y cuestiones prácticas en el momento de la planificación de una red inalámbrica. Existen una serie de herramientas como inSSIDer, NetSurveyor, NetSpot y Vistumbler orientados a la planificación y diseño de redes LAN inalámbricas $802.11 \mathrm{a} / \mathrm{b} / \mathrm{g} / \mathrm{h} / \mathrm{ac}$ en la obtención de rendimiento, seguridad y cumplimiento óptimo de la red. Los resultados obtenidos mediante experimentación permiten obtener información relevante para el planificador de la red encargado de diseñar e implementar la red LAN inalámbrica. De igual modo, se hace uso de una serie de escenarios para la simulación, los cuales están configurados con ciertas características, lo que permite la verificación de la velocidad de conexión, frecuencia de la señal y capacidad de transmisión afrontando las etapas de congestión de la red.

Palabras clave: redes de áreas locales inalámbricas, planificación inalámbrica, localización de los puntos de acceso, escenarios internos, herramientas de planificación, diseño de redes inalámbricas Lan.

Title: Planning in wireless local area networks in indoor scenarios: elements, tools and practical issues.

\footnotetext{
Abstract: Today, some of the difficulties that wireless networks of local area (WLAN) have in internal spaces, for example, buildings, offices, distribution of cubicles between floors, is the adequate coverage that has access to various types of artifacts such as cell phones, laptops, computers, through the wireless connection that provides the various access points (AP) distributed in various spaces where people walk or work.
}

In this article, we present an analysis of the internal scenarios, considering a series of basic elements, characteristics, tools and practical issues at the time of the planning of a wireless network. There are a number of tools such as inSSIDer, NetSurveyor, NetSpot and Vistumbler oriented to the planning and design of wireless LAN 802.11 a / b / g / $\mathrm{n} /$ ac in obtaining performance, security and optimal compliance of the network.

The results obtained through experimentation allows obtaining relevant information for the network planner responsible for designing and implementing the wireless LAN. Similarly, it makes use of a series of scenarios for the simulation, which are configured with certain characteristics, which allows the verification of the connection speed, frequency of the signal and transmission capacity facing the stages of network congestion.

Key words: wireless local area networks, wireless planning, locate of the access point, indoor scenarios, planning tools, design of wireless LAN networks.

Tipo de artículo: tutorial

Fecha de recepción: 14 de mayo de 2018

Fecha de aceptación: 17 de octubre de 2018

\section{Introducción}

Es una realidad que las Redes de Área Local Inalámbricas (WLAN) han venido a sustituir espacios en laboratorios, oficinas, centros comerciales, centros hospitalarios; donde antes existía cableado estructurado específicamente bajo el estándar ANSI/TIA/EIA-568A. Lo anterior conlleva a que los diseñadores y planificadores en redes WLAN específicamente en escenarios internos tengan que replantear la manera en cómo se van a comunicar y ubicar los equipos sean estos las computadoras, puntos de acceso, antenas, por mencionar solo algunos. Sin embargo, una planificación adecuada de la red inalámbrica va más allá de proporcionar una cierta capacidad a los usuarios [25]. A su vez, la función integral de estos artefactos depende en gran manera de una correcta planificación de distribución de las frecuencias y la calidad en la cobertura según comentan [1], [2] que puedan brindar los puntos de acceso 0 estaciones bases dependiendo de su ubicación. Sin embargo [3], [4] señalan que la planificación de las redes de área local inalámbricas enfrenta dos grandes problemas. El primero, es la ubicación de los puntos de acceso, como se aprecia en la figura 1, y segundo, la distribución de frecuencias de la señal en las estaciones base o puntos de acceso.

En la figura 1, se observa la distribución de tres puntos de acceso A, B y C. Sin embargo, estos puntos de acceso (AP) están distribuidos en un espacio determinado por lo que el diseñador de la red debe considerar los sitios que están identificados en las esquinas en ambientes interiores, por ejemplo, en una oficina. Los puntos de acceso (AP - A y AP - B), emiten una frecuencia de señal en forma simultánea que genera colisión con la señal que emite la estación base identificada como AP-C. Frecuentemente, una adecuada planificación de una WLAN requiere no solo conocer los estándares $802.11 \mathrm{a}, \mathrm{b}, \mathrm{g}, \mathrm{n}$, an, sino que también se 
debe seleccionar el tipo de escenario para una oficina u hotel, escenarios en espacio abierto mejor conocido como outdoor scenarios que requieren los centros hospitalarios, cafeterías, metros y los ambientes comerciales (aeropuertos, almacenes de datos que sirven como espacios para guardar todo tipo de enceres que ya no se usan) en el cual, se va a llevar a cabo la planificación de la red.

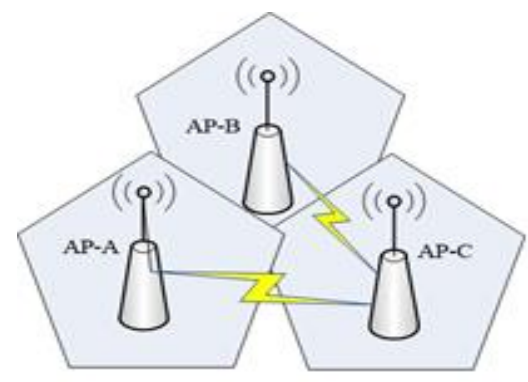

Figura 1. Problema de la ubicación y distribución de frecuencias de la señal en los puntos de acceso.

Por otro lado, encontramos en algunas investigaciones [5], [6] donde exponen que la selección de los sitios forma la base de una red la cual debe cumplir con una serie de requerimientos, como una amplia cobertura, alta capacidad de tráfico, pero que permita minimizar el costo de inversión de la infraestructura tecnológica. Sin embargo [7], [8], [11] explican que el planeamiento apropiado de una red es en algunas situaciones necesario para adquirir una adecuada cobertura.

Por lo tanto, la planificación de infraestructura en redes de área local inalámbricas (WLAN) que se oferta en grandes edificios requiere de la consideración de aspectos relacionados con la cobertura, densidades de tráfico, interferencias y costos de minimización y por lo que es una tarea difícil si es hecha manualmente [9]. De igual modo, el alto nivel de heterogeneidad y la falta de estandarización en todas las tecnologías, hacen que el diseño de dichos entornos sea una tarea muy desafiante, ya que cada instalación debe diseñarse manualmente y realizarse ad-hoc para el edificio específico [30]. Por eso, la localización de los puntos de acceso [26], el comportamiento de los puntos de acceso, la orientación de las antenas, la emisión de energía [29] y el canal de frecuencia de la antena [10], representa una dificultad al momento de parametrizar estas variables que son necesarias en el planeamiento automático de este tipo de redes.

En este artículo, los resultados y las contribuciones alcanzados en el análisis de las redes WLAN en escenarios internos, al utilizar una serie de herramienta, permite la optimización de la infraestructura tecnológica y un diseño y planificación de este tipo de redes inalámbricas, las cuales se detallan en el apartado 2.3 de este estudio.

La organización de este artículo es la siguiente. En la sección 2 , métodos y materiales, hace referencia a los insumos utilizados en la elaboración de este artículo. A su vez, la sección 3, resultados y discusión, se ilustra el análisis de una planificación
WLAN a través de la diversidad de herramientas que existen para la planificación y diseño de redes LAN inalámbricas. Por último, se explican en la sección 4 las consideraciones finales y detallan las referencias utilizadas en este artículo.

\section{Métodos y materiales}

Se lleva a cabo un proceso sistémico que permite la planificación y revisión de la documentación a partir de las distintas posiciones propuestas por cada uno de los diferentes autores.

Se investiga acerca de trabajos realizados por estos autores [14 - 20], donde se explora lo relacionado al ámbito de la planificación en redes de área local inalámbricas para confrontar escenarios internos con las características propias de estos ambientes. Sin embargo, se considera los tipos de ambientes e informes, trayectoria de propagación [21], ubicación de puntos de acceso, pero enfocado a las redes de área local inalámbricas (WLAN) con el fin de establecer un punto de partida. Se contempla los trabajos que tratan el concepto de planificación en redes WLAN, pero específicamente en ambientes o escenarios internos, así como el nivel de ruido, promedio de la relación señal/ruido (SNR), interferencias de canales adyacentes, modos de operación de los puntos de acceso, ancho del canal, mapas de calor, utilizadas para abordar el tema de la planificación en redes inalámbricas las cuales son varias, tomando en cuenta el criterio de cada autor.

Las revistas especializadas tomadas en cuenta desde una perspectiva de fuente primaria son las relacionadas con la IEEE, ACM, Elsevier, Springer, entre otras.

\subsection{Métodos matemáticos para la localización inalámbrica basada en escenarios internos}

En este trabajo de investigaciónón, se toma como referencias los diversos métodos matemáticos utilizados para las localizaciones inalámbricas basadas en escenarios [12] expuestas por el autor Junjie, entre las que destacan la proximidad, la triangulación y fingerprint (huella dactilar). De estas, se utiliza la denominada triangulación, en la que diversos autores y expertos la consideran como la técnica más común no basada en estudios [27], al permitir medir la distancia entre el terminal móvil y tres transmisores colocados en diferentes posiciones, obteniendo la estimación de la ubicación mediante la resolución de funciones algebraicas, ya que también, utiliza aspectos teóricos provenientes de la geometría para obtener la localización de un usuario al estar determinado para cada una de las distancias que son asignadas a los puntos de medición 0 puntos de acceso. Claramente, las técnicas no basadas en la investigación están limitadas por el modelo de pérdida de ruta. Desafortunadamente, un modelo de pérdida de ruta precisa y universal es difícil de lograr en un ambiente interior complejo y variable [28].

La figura 2, muestra cómo obtener la localización de un usuario a través de la distancia, en la cual se tienen tres puntos de acceso inalámbricos $A, B$ y $C$, respectivamente, ubicados en posiciones fijas. Si la distancia del punto del usuario con respecto 
a las tres bases de acceso inalámbrico es conocida, entonces, la localización del punto de acceso del usuario puede ser expuesta como la intersección de los tres círculos.

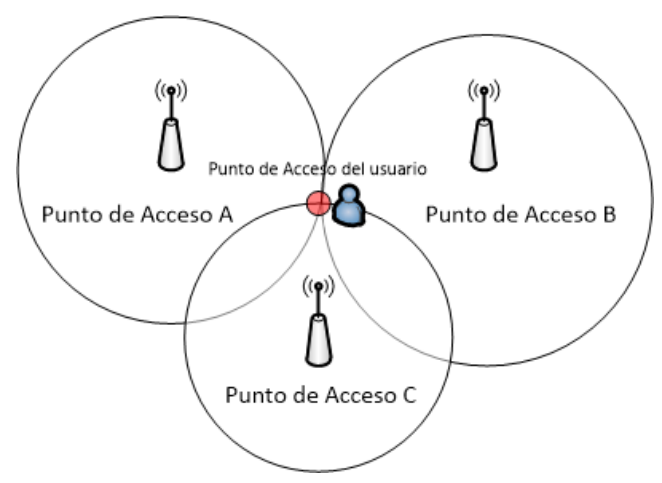

Figura 2. Localización de un usuario a través de la distancia.

Entonces, la localización de un usuario a través de la distancia de los puntos de acceso se expresa mediante la siguiente ecuación. En la ecuación "(1)", la $d$, es la distancia entre los puntos de acceso, mientras que la $\Omega$, hace referencia al punto de acceso del usuario y la Ls, es la distancia de separación en dónde se encuentre ubicados esos puntos de acceso, por ejemplo, la pared, en el techo...etc.

$$
d=\Omega . \text { Ls }
$$

Sin embargo, también se puede obtener la ubicación del usuario si el ángulo de las estaciones base para el punto del usuario en relación a las estaciones base es conocido, como se observa en la figura 3, al permitir así, obtener la localización del punto de usuario mediante la intersección de los tres vectores.

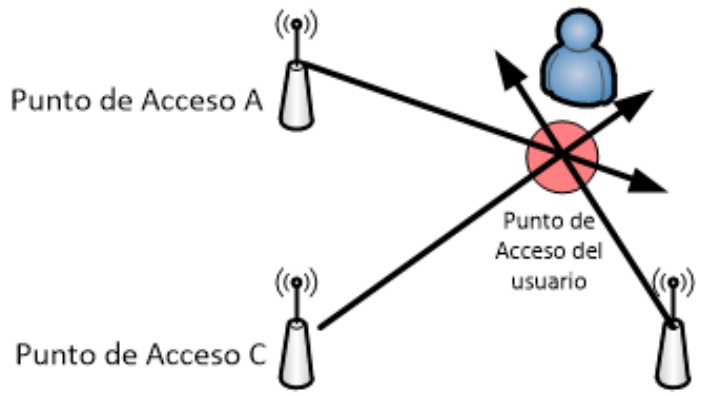

Punto de Acceso B

Figura 3. Localización de un usuario a través de la intersección de los tres vectores.

\subsection{Localización en escenarios internos basados en WiFi}

Una de las ventajas de usar sistemas de posicionamiento WiFi es localizar la ubicación de la mayoría de los dispositivos compatibles con WiFi sin instalar software adicional ni realizar cambios adicionales al hardware. Sin embargo, en ambientes WLAN, no se requiere línea de visión. Debido a esta ventaja, los sistemas de posicionamiento WiFi se han convertido en el enfoque más extendido para la localización de interiores [22].

La mayoría de los sistemas de posicionamiento basados en WiFi (WLAN) están disponibles en forma de productos comerciales a nivel de prototipos basados en mediciones de la intensidad de la señal recibida (RSS). De igual modo, los sistemas de posicionamiento basados en WiFi tienen varias ventajas [23]. En primer lugar, en términos de costo, la implementación de las infraestructuras WLAN de los algoritmos de posición no requiere hardware adicional, ya que las tarjetas de interfaz de red (NIC) miden los valores de intensidad de señal de todos los puntos de acceso inalámbrico a nivel de alcance del receptor. Por lo tanto, las señales necesarias para el posicionamiento se pueden obtener directamente desde NIC disponibles en la mayoría de los dispositivos de computación de mano. Debido a la ubicuidad de las WLAN, este modo de posicionamiento proporciona una solución particularmente rentable para ofrecer LBS (Servicios Basados en Localización) en escenarios internos de centros comerciales y residenciales [24]. En segundo lugar, los sistemas de posicionamiento WLAN ofrecen escalabilidad en dos aspectos: primero sin costo en los requisitos de infraestructura y hardware, segundo, la cantidad de dispositivos móviles que se suscriben a los servicios de posicionamiento. De este modo, también hay ciertas limitaciones de WLAN: atenuación de la señal del entorno estático, como la pared, el movimiento de los muebles y las puertas. Algunas de las fortalezas, debilidades y oportunidades de WiFi se presentan en la tabla 1. [23]

Tabla 1. Fortalezas, debilidades y oportunidades de WiFi

\begin{tabular}{|c|c|c|}
\hline Fortalezas & Debilidades & Oportunidades \\
\hline $\begin{array}{l}\text { Se encuentra en } \\
\text { todos los edificios, } \\
\text { una buena } \\
\text { potencia de señal } \\
\text { disponible. }\end{array}$ & $\begin{array}{l}\text { La puesta a punto de } \\
\text { lugar requiere mucho } \\
\text { tiempo y un considerable } \\
\text { uso de mano de obra. }\end{array}$ & $\begin{array}{llr}\text { Los fingerprints no } \\
\text { necesitas } & \text { estudios } \\
\text { geométricos. } & \end{array}$ \\
\hline $\begin{array}{l}\text { Las señales WiFi } \\
\text { pueden penetrar } \\
\text { las paredes } \\
\text { donde falla el } \\
\text { GPS. }\end{array}$ & $\begin{array}{l}\text { Multitrayecto influenciado } \\
\text { por la presencia de } \\
\text { objetos físicos. }\end{array}$ & $\begin{array}{l}\text { Los "fingerprints" solo es } \\
\text { necesaria en lugares } \\
\text { seleccionados. }\end{array}$ \\
\hline $\begin{array}{l}\text { Ubicación de los } \\
\text { fingerprints en } \\
\text { lugares } \\
\text { disponibles. } \\
\end{array}$ & $\begin{array}{l}\text { La intensidad de la señal } \\
\text { cambia de acuerdo con } \\
\text { las variaciones que se } \\
\text { producen en el tiempo. }\end{array}$ & \\
\hline & $\begin{array}{l}\text { Interfiere con otros } \\
\text { dispositivos en el ISM } \\
\text { (bandas reservadas para } \\
\text { la industria, lo cientifico y } \\
\text { la medicina) de } 2,4 \mathrm{GHz} \text {. }\end{array}$ & \\
\hline
\end{tabular}

\subsection{Herramientas de planificación y diseño de redes LAN inalámbricas}

En efecto y para este estudio en particular se utilizaron diversas herramientas de planificación y diseño de redes LAN inalámbricas, unas de software libre y otras con licencia de pago, 
debido a que los parámetros utilizados en el proceso de medición experimental no están contemplados en una sola herramienta.

Por lo tanto, se utilizó la herramienta de monitoreo inSSIDer versión 2.1 [13] para analizar el comportamiento de la señal en cada uno de los APs, como se observa en la figura 5.

De igual modo, se tuvo presente la herramienta NetSurveyor 802.11 Discovery Tool [31], para analizar el tipo de canal, el beacon y la calidad de la señal (se hizo también de Vistumbler v10.6.5 para medir la calidad de la señal en porcentajes (\%)), emitidos por cada uno de los APs como se observa en la tabla 2. También, podemos observar en la figura 6 , la relación entre los tipos de canal y la suma de los porcentajes de calidad de los beacon.

Con la herramienta Vistumbler v10.6.5 [32], se analizaron el tipo de autenticación, tipo de encriptación, tipo de infraestructura, tipo de radio, y promedios de transferencias básica, como se observa en la tabla 3.

Otros parámetros que se consideraron para cada uno de los APs y que han sido analizados con la herramienta NetSpot Discovery [33], guardan relación con el porcentaje (\%), mínimos, máximos, promedio y banda de la señal. Esto se observa en la tabla 4.

\section{Resultados y discusión}

En el planteamiento de la planificación de una red de área local inalámbrica (WLAN), específicamente en escenarios internos se hace uso de un mapa visual de las conexiones con Wi-Fi digitalizado cuyas dimensiones son: $39.51 \mathrm{~m}$ x $39.76 \mathrm{~m}$, en una escala de 1:100. En la figura 4, se identifican tres secciones denominadas AP-A, AP-B y AP-C que hacen referencia a tres puntos de acceso (AP).

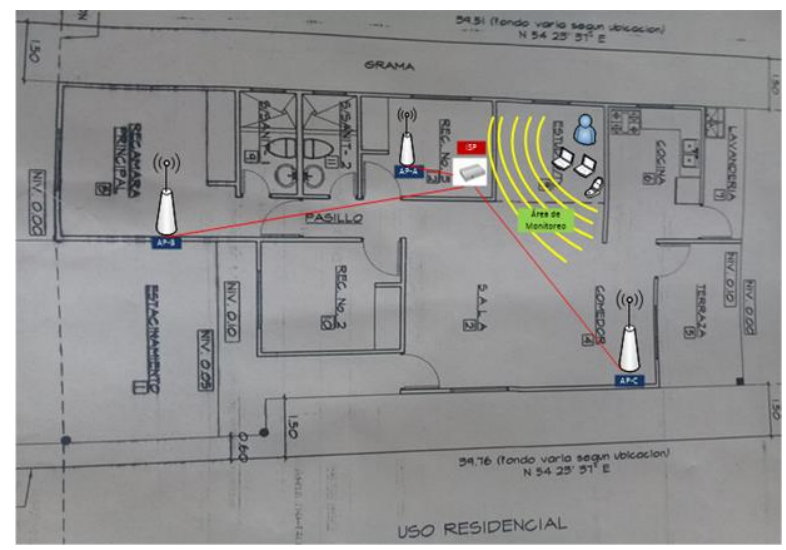

Figura 4. Mapa visual de la conexión Wi-Fi.

El escenario real utilizado, es una casa que tiene en construcción $166.68 \mathrm{~m}$ cuadrados de un total de $315 \mathrm{~m}$ cuadrados. En esta casa, el AP_A, se encuentra ubicado en la recámara $N^{\circ} 1$, junto al ISP, mientras que el AP_B se encuentra en la recámara principal y el AP_C se localiza en la sección denominada sala-comedor.

Cada uno de estos puntos de acceso tiene un puerto WAN que está conectado al ISP (Proveedor de Servicios de Internet) a través de un puerto RJ-45 del ISP. A su vez, en la fase de experimentación se hacen uso de dos Laptops, una Lenovo Intel(R) Celeron(R) $3205 \mathrm{u}, 1.50 \mathrm{GHz}$ con un adaptador de red inalámbrico Qualcomm Atheros AR956x y otra VAIO Sony AMD $\mathrm{E}-350,1.60 \mathrm{GHz}$, ambas con $4 \mathrm{~GB}$ de memoria para verificar el acceso a red inalámbrica, monitorear el comportamiento de cada uno de los AP a nivel de canales, comportamiento de la señal, tipo seguridad, mac address, velocidad promedio (mínima y máxima), tipo de red, entre otros aspectos relevantes. También se hizo uso de un celular Blu y una Tableta genérica para comprobar el acceso a la red Internet.

Además, se utilizó cableado estructurado del tipo Cat5e, con diferentes longitudes de cables, por ejemplo: 13.5 metros, 1 metro y 9.25 metros para conectar cada uno de los puntos de acceso al ISP.

Por otro lado, las características de los routers inalámbricos utilizados en procese de experimentación se detallan en la tabla 2.

Tabla 2. Características de los routers inalámbricos

\begin{tabular}{|c|c|c|c|c|c|}
\hline $\begin{array}{c}\text { Nombre } \\
\text { AP }\end{array}$ & Marca & Modelo & $\begin{array}{l}\text { Dirección } \\
\text { MAC }\end{array}$ & $\begin{array}{l}\text { Número de } \\
\text { Antenas }\end{array}$ & $\begin{array}{c}\text { Distancia en } \\
\text { metros }(\mathrm{m}) \mathrm{y} \\
\text { centimetros }(\mathrm{cm}) \\
\text { con respecto al } \\
\text { ISP } \\
\end{array}$ \\
\hline AP_A & $\begin{array}{c}\text { Nexx } \\
t \\
\text { Solut } \\
\text { ions } \\
\end{array}$ & $\begin{array}{c}\text { ARNO230 } \\
4 \mathrm{u} 2\end{array}$ & $\begin{array}{c}\text { C83A352 } \\
\text { 3ADF0 }\end{array}$ & 2 & $40 \mathrm{~cm}$ \\
\hline$A P_{-} B$ & $\begin{array}{l}\text { TP- } \\
\text { Link }\end{array}$ & $\begin{array}{c}\text { TL- } \\
\text { WR940N }\end{array}$ & $\begin{array}{c}\text { 90F65297 } \\
\text { D1DE }\end{array}$ & 3 & $6 \mathrm{~m}$ \\
\hline AP_C & $\begin{array}{l}\text { Nexx } \\
t \\
\text { Solut } \\
\text { ions }\end{array}$ & $\begin{array}{c}\text { ARN0230 } \\
4 \text { u8 }\end{array}$ & $\begin{array}{c}\text { CO25675 } \\
\text { 893AC }\end{array}$ & 3 & $7 \mathrm{~m}$ \\
\hline
\end{tabular}

Sin embargo, a nivel del comportamiento de la señal en cada uno de los puntos de acceso se llevó a cabo una comparación de entre los mismos. Los resultados obtenidos se muestran en la figura 5.

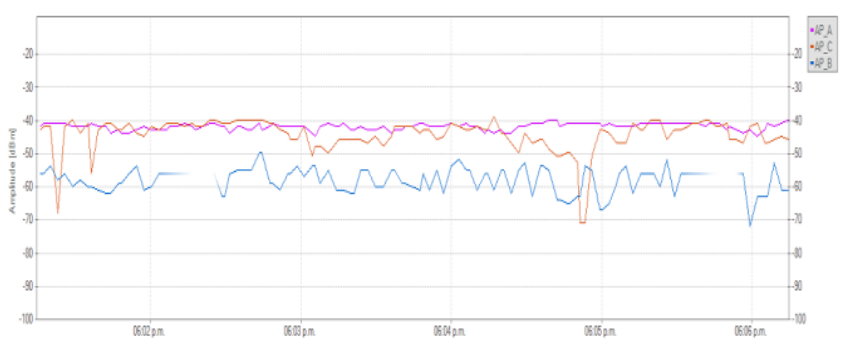

Figura 5. Comportamiento de la señal en los APs.

En la figura 5 , tenemos una gráfica de tiempo vs. amplitud ( $\mathrm{dBm})$ y se observa que el AP_A, en color morado es más estable con respecto al $A P \_C$, y $A P \_B$, respectivamente. De igual modo, la señal en el AP_C, en color rojo sufre de mayores degradaciones debido a las interferencias con el AP_A. Sin embargo, en AP_B, en color azul celeste, existen periodos de tiempo donde la señal 
se degrada en su totalidad y se pierde conectividad, lo que hace de la señal muy inestable por momentos.

A su vez, se monitorizaron otros parámetros relevantes, por ejemplo, tipo de canal, tipo de marco de baliza o beacon que son tramas que contienen toda la información de la red y se transmiten periódicamente por medio del AP en un conjunto básico de infraestructura de servicios (BSS) y sirven para anunciar la presencia de una LAN inalámbrica relacionados con cada uno de los puntos de acceso, como se observa en la tabla 3.

Tabla 3. Canal, beacon y calidad de la señal

\begin{tabular}{|c|c|c|c|c|c|}
\hline $\begin{array}{c}\text { Nombre } \\
\text { AP }\end{array}$ & Canal & $\begin{array}{c}\text { Longitud del } \\
\text { beacon } \\
(\mathrm{dBm})\end{array}$ & $\begin{array}{c}\text { Longitud del } \\
\text { beacon } \\
\left(\mathrm{mWatt} \times 10^{\circ} 6\right)\end{array}$ & $\begin{array}{c}\text { Calidad del } \\
\text { beacon } \\
(\%)\end{array}$ & $\begin{array}{c}\text { Calidad } \\
\text { de la } \\
\text { señal }\end{array}$ \\
\hline AP_A & 6 & -48 & 12.5893 & 73 & Excelente \\
\hline AP_B & 4 & -63 & 1.9953 & 46 & Muy bueno \\
\hline AP_C & 6 & -39 & 158.4825 & 76 & Excelente \\
\hline
\end{tabular}

Se observa en la tabla 2, que los AP_A y AP_C, tiene mejor calidad en la señal de un $100 \%$, con respecto al $A P \_B$ de un $92 \%$. Este último tiene una calidad del beacon del $46 \%$ con respecto al $76 \%$ del AP_C y un $73 \%$ del AP_B. En la figura 6, podemos observar una comparativa entre los canales utilizados por los APs y suma de porcentajes de la calidad de los beacons.

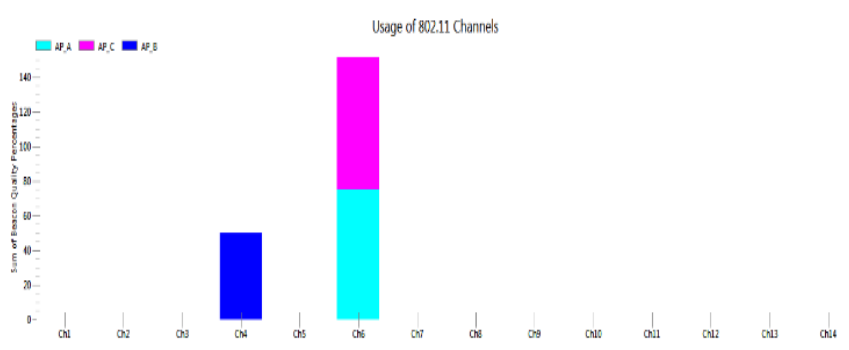

Figura 6. Canales vs. Suma de porcentajes de la calidad de los beacons.

De esta forma se analizaron para cada uno de los APs, los tipos de autenticación, encriptación, infraestructura, radio y promedios de transferencia básica, como se observa en la tabla 4.

Tabla 4. Tipos de autenticación, encriptación, infraestructura, radio y promedios de transferencia básica

\begin{tabular}{|c|c|c|c|c|c|}
\hline $\begin{array}{c}\text { Nombre } \\
\text { AP }\end{array}$ & $\begin{array}{c}\text { Autenti- } \\
\text { cación }\end{array}$ & $\begin{array}{c}\text { Encripta- } \\
\text { ción }\end{array}$ & $\begin{array}{c}\text { Infraestruc- } \\
\text { tura }\end{array}$ & Radio & $\begin{array}{c}\text { Promedio de } \\
\text { transferencia } \\
\text { básica }\end{array}$ \\
\hline AP_A & WPA & CCMP & $\begin{array}{c}\text { Infraestruct } \\
\text { ura }\end{array}$ & $\begin{array}{c}802.1 \\
1 \mathrm{n}\end{array}$ & $\begin{array}{c}1,2,5.5, \\
11,24,36, \\
54\end{array}$ \\
\hline AP_B & WPA2 & CCMP & $\begin{array}{c}\text { Infraestruct } \\
\text { ura }\end{array}$ & $\begin{array}{c}802.1 \\
1 \mathrm{n}\end{array}$ & $\begin{array}{c}1,2,5.5, \\
11\end{array}$ \\
\hline AP_C & WPA2 & CCMP & $\begin{array}{c}\text { Infraestruct } \\
\text { ura }\end{array}$ & $\begin{array}{c}802.1 \\
1 \mathrm{n}\end{array}$ & $\begin{array}{c}1,2,5.5, \\
11\end{array}$ \\
\hline
\end{tabular}

También, se analizaron otros parámetros relacionados con la señal de cada uno de los APs, entre los que destacan los porcentajes (\%), mínimos, máximos, promedios y banda base. Los resultados de observan en la tabla 5.

Tabla 5. Parámetros relacionados con la señal

\begin{tabular}{|c|c|c|c|c|c|}
\hline $\begin{array}{c}\text { Nombre } \\
\text { AP }\end{array}$ & $\begin{array}{c}\text { Porcentaje } \\
(\%)\end{array}$ & Mínimos & Máximos & Promedio & $\begin{array}{c}\text { Banda } \\
\text { Base (GHz) }\end{array}$ \\
\hline AP_A & 64 & -68 & -33 & -40 & 2.4 \\
\hline AP_B & 41 & -96 & -34 & -61 & 2.4 \\
\hline AP_C & 66 & -49 & -35 & -42 & 2.4 \\
\hline
\end{tabular}

Se observa, en la tabla 5 , que los mejores porcentajes en señal lo tienen los AP_C (66\%) y AP_A (64\%), respecto a AP_B con un $41 \%$. En promedio el AP_A (-40) y el AP_C (-42), serían los mejores puntos para conectarse a la Internet con respecto al AP_B (-61).

\section{Conclusión}

En este artículo la planificación con respecto a cómo van a ser distribuidos los puntos de acceso en una red inalámbrica local en escenarios internos, adquiere mayor relevancia debido a que se requiere por parte del planificador de la red una mayor precisión en el momento de ubicar estos puntos de acceso para que no se generen colisiones entre las frecuencias y puntos muertos dónde la señal no llega. Lo novedoso en estos procesos de planificación, es que se cuentan con una serie de herramientas de planificación y diseño de redes LAN inalámbricas, utilizadas en la fase de experimentación y en este estudio entre las que destacan inSSIDer 2.1, NetSurveyor 802.11 Discovery Tool, NetSpot Discover y Vistumbler v10.6.5 que permiten analizar el comportamiento de ciertos elementos relacionados con el análisis del comportamiento de la señal, tipos de canal, beacon, calidad de la señal, tipos de autenticación, encriptación, infraestructura, radio, promedios de transferencia básica, transferencia de paquetes y cuestiones prácticas que guardan relación con el nivel de cobertura de la frecuencia de cada uno de estos puntos de acceso en un ambiente cerrado. Todo lo anterior, ayuda a tener una mejor perspectiva y toma de decisión al momento de llevar a cabo una planificación de redes WLAN, en escenarios internos por parte del constructor y diseñador de la red.

En la fase de experimentación, los AP_A y AP_C fueron los que mejor resultados tuvieron con respecto a la calidad de la señal, un $100 \%$, con respecto al AP_B de un $92 \%$. Sin embargo, el AP_B sufre de fuertes caídas en la señal, debido a su ubicación con respecto al IPS, ya que entre ambos se encuentran ubicados 2 baños, lo que hace que la calidad de la señal se degrade y su cobertura no sea la más satisfactoria. Inclusive, el AP_B se reubico en la recámara $N^{\circ}{ }^{\circ}$ pero provoca que la señal del AP_C se traslape con la señal del AP_A provocando una degradación e interferencias con la señal del AP_C. 
Dada la situación anterior, lo recomendable es que se deje el AP_C en la sala-comedor y el AP_A en recámara $\mathrm{N} .{ }^{\circ} 1$, para una mejor cobertura de la señal Wi-Fi. Es importante considerar el espacio donde se ubica el AP, que las paredes tengan el mínimo de obstáculos posibles a nivel de cubículos, la calidad en el cableado estructurado que no esté corrugado, ángulos que se conforman entre las paredes, entre otros aspectos relevantes. Para próximo estudio, es recomendable utilizar un medidor de señales Wi-Fi, por ejemplo, un dispositivo como WifiMETRIX que ayudaría a medir de señal en diversos canales y a localizar puntos muertos, independientemente de la herramienta de planificación y diseño de redes LAN inalámbricas que se vaya a utilizar.

Como líneas de investigación, se plantea el implementar este mismo tipo de escenarios en un entorno de Sistema Operativo de Red Abierta como es ONOS y los emuladores Mininet y MininetWiFi, este último muy útil para este tipo de escenario de redes LAN inalámbricas. Se utilizaría como protocolo de comunicación OF (Open Flow).

\section{Referencias}

[1] A. Farsi, N. Achir \& Boussetta, K. Ann. WLAN planning: Separate and joint optimization of both access point placement and channel assignment. Telecommunications. 2015, 70(5): p. $263-274$.

[2] Y-K. Lin, Y-C. Tsao, S-W, Lin. Wireless LAN Access Point Location Planning In: Proceedings of the institute of industrial engineers Asian conference 2013. Springer, Singapore. 2013, 1(1): p. $907-914$.

[3] K. Farkas, Á. Huszák, et al. Optimization of Wi-Fi Access Point Placement for Indoor Localization. Informatics \& It Today, 2013, 1(1): p.28-33.

[4] Á. Huszák, G. Gódor, et al. On the Placement of Wi-Fi Access Points for Indoor Localization. En: 1st Global Virtual Conference. 19. Information Technology. Hungary: EDIS - Publishing Institution of the University of Zilina, 2013, p. 8- 12.

[5] R.Henniger .Current approaches of Wifi Positioning. Service - Centric Networking - Seminar WS 2011/2012, 2012, p. 1-8.

[6] B. Bellalta, L. Bononi, R.Bruno, et al. Next generation IEEE 802.11 Wireless Local Area Networks: Current status, future directions and open challenges. Computer Communications, 2015, 23(37): p. 1-25.

[7] Z. Farid, R. Nordin, et al. Recent Advances in Wireless Indoor Localization Techniques and System. Journal of Computer Network and Communications, 2013, 2013 (2013): p. 1 - 12.

[8] N. Liu, D. Plets , K. Vanhecke, L. Martens, et al. Wireless indoor network planning for advanced exposure and installation cost minimization. EURASIP Journal on Wireless Communications and Networking, 2015, 2015(199): p. 1-14.

[9] P. Wertz, M. Sauter, M. Landstofer et al. Automatic Optimization Algorithms for the Planning of Wireless Local Area Networks, Vehicular Technology Conference, 2004 IEEE, Vol. 4, pp. 3010 - 3014.

[10] A. Gondran, A. Caminada, J. Fondrevelle and O. Boala. Wireless LAN planning: a didactical model to optimise the cost and effective payback, Int. J. Mobile Networking Desing and Innovation, Vol. 2. No.1, pp. 13-25, 2007.

[11] Lu. Jia-Liang, F.Valois. Performance evaluation of 802.11 WLAN in a real indoor environment, Wireless and Mobile Computing Networking and Communications, 2006. (WiMob'2006). IEEE Internacional Conference on, vol. no. , pp. 140 - 147, 19 - 21, June 2006.

[12] L. Junjie. Survey of Wireless Based Indoor Localization Technologies. http://www.cse.wustl.edu/ jain/cse574-14/ftp/indoor/index.html, April 30,2014, pp.1-17

[13] InSSIDer. https://www.metageek.com/products/inssider/
[14] B. Wenche et. al. WLAN network planning and setup - Best Practice Document. TERENA, December 2010. https://www.terena.org/campus-bp/

[15] V.Honkavirta, T. Perälä, S. Ali-Löytty, R. Piché. A comparative survey of WLAN location fingerprinting methods. Proceedings of the 6th Workshop on Positioning, Navigation and Communication 2009 WPNC'09, March 19, 2009, Hannover, Germany pp. 243-251.

[16] L. Youngseok, K. Kyongae, and Ch. Yanghee. Optimization of AP Placement and Channel Assigmente in Wireless LANs. Proceedings of the 22th Annual IEEE. Conference on Local Computer Network (LCN'02), IEEE Computer Society, 2002, pp. 1-6.

[17] S. Selena, J.M., Louis David, H.Jeffrey. Landscape of connectivity. Measuring and representing fluctuations in wireless network traffic in space.Automation in Construction, Elsevier, May 2018, pp. 1 - 17.

[18] X. Yalong, Z. Shigeng, C.Jiannong, W. Haodong, W. Jianxin, Exploiting Distribution of Channel State Information for Accurate Wireless Indoor Localization, Computer Communications (2017), doi: 10.1016/j.comcom.2017.10.013

[19] S. Stephen. Network Planning with a Performance - Prediction Tool. Internacional Journal of Network Management, 9, 1999, pp. 167 - 173

[20] R.C. Rodrigues, G.R. Mateus, A.A.F. Loureino. On the design and capacity planning of a Wireless local area network, Network Operations and Management Symposium, 2000. NOMS 2000. 2000 IEEE/IFIP, pp.335-348.

[21] Z. Lei, R.P. José, R.O.F. Jean, A.G.N. José, W.M. David, B.Cesar, C. Luis. Propagation modeling for outdoor - to - indoor and indoor - to - indoor wireless links in high - speed train. Measurement, Elsevier, Vol. 100, November 2017, pp. $43-52$.

[22] M. B. Ismail, A. F. A. Boud, andW. N.W. Ibrahim, "Implementation of location determination in a wireless local area network (WLAN) environment," in Proceedings of the 10th International Conference on Advanced Communication Technology (ICACT '34), pp. 894-899, February 2008.

[23] F. Zariel, N. Rosdiadee, and I. Moham. Recent Advances on Wireless Indoor Localization Techniques and System. Hindawi Publishing Corporation, Journal of Computer Networks and Communications, Volume 2013, 12 pages, doi /10115/2013/185138.

[24] C.Marc, M. E. Israel, and B.A. Francisco, "Location in wireless local area networks," in Location-Based Services Handbook, pp.67-90, CRC Press, 2010.

[25] L. Ning, P.Dariel, V.Kris, M. Lue and J.Wout. Wirelss indoor networking planning for advanced exposure and installation cost minimization. EURASIP Journal on Wireless Communications and Networking, 2015, 2015:199, DOI 10.1186/s 13638-015-0425-Z.

[26] D. Liang, Z. Zhang and M. Peng, "Access Point Reselection and Adaptive Cluster Splitting-Based Indoor Localization in Wireless Local Area Networks," in IEEE Internet of Things Journal, vol. 2, no. 6, pp. 573-585, Dec. 2015.

[27] M. Ren and Y. Zou, "A novel multiple sparse source localization using triangular pyramid microphone array," IEEE Signal Processing Letters, vol. 19, no. 2, pp. 83-86, Feb. 2012.

[28] M. Peng, D. Liang, Y. Wei, J. Li, and H. Chen, "Self-configuration and selfoptimization in LTE-Advanced heterogeneous networks," IEEE Communications Magazine, vol. 51, no. 5, pp. 36-45, May 2013.

[29] A. Gureev, V. I. Selivantsev, T. Tun and Y. Pavlyuk, "Using experimental data for indoor wireless system planning," 2018 IEEE Conference of Russian Young Researchers in Electrical and Electronic Engineering (ElConRus), Moscow, 2018, pp. 1731-1733.

[30] A. Cirigliano, R. Cordone, A. A. Nacci and M. D. Santambrogio, "Toward Smart Building Design Automation: Extensible CAD Framework for Indoor Localization Systems Deployment," in IEEE Transactions on Computer-Aided Design of Integrated Circuits and Systems, vol. 37, no. 1, pp. 133-145, Jan. 2018.

[31] NetSurveyor. http://nutsaboutnets.com/

[32] Vistumbler. https://www.vistumbler.net/

[33] NetSpot. https://www.netspotapp.com/ 